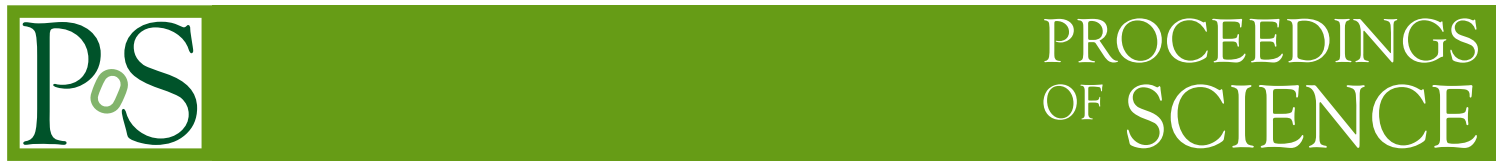

\title{
Investigations of hadronization process in DIS at CLAS
}

\author{
A. Daniel* and K. Hicks \\ Department of Physics and Astronomy, Ohio University, Athens, Ohio, 45701, USA \\ E-mail: adanielejlab.org
}

Hadronization is the process through which partons, created in an elementary reaction, turn into hadrons. This phenomena has been studied for decades, however, the space-time features of this fundamentally non-perturbative process is not clearly understood. Semi-inclusive measurements of deep inelastic electron scattering from nuclei provide a unique testing ground to study the process of hadron formation. The space-time features and the nuclear dependence of quark propagation and hadronization can be extracted by comparing the production of various hadronic species from a number of target nuclei under different kinematic conditions. The CLAS experiment E02-104 was run with a variety of nuclear targets using a $5 \mathrm{GeV}$ beam energy. The goal of this experiment is to measure observables related to propagation of a quark through cold nuclear matter.

XVIII International Workshop on Deep-Inelastic Scattering and Related Subjects

April 19 -23, 2010

Convitto della Calza, Firenze, Italy

\footnotetext{
* Speaker.
} 


\section{Introduction}

The study of hadronization, the process in which partons produced in some elementary interaction evolves to the hadrons, is not well understood. The hadron formation is dominated by non perturbative process and cannot be approached with the help of perturbative QCD. For that reason various types of models have been developed to understand the dynamics of hadronization process. Phenomenological models use formation times and absorption cross sections for the various hadrons in the nuclear medium. Another category of (QCD inspired) models focus on the energy loss due to gluon radiation that the struck quark experiences in the nuclear environment. A recent review on parton propagation can be found in reference [1]. One of the main goals discussed here using data from CLAS is to reach a better understanding of in-medium hadronization in order to provide a more reliable interpretation of the high $p_{T}$ hadron production in relativistic heavy ion collisions. Also, it is useful obtain a good understanding of the hadron modifications in the nuclear medium for the proper interpretation of the neutrino oscillation experiments since these experiments use nuclear targets to enhance the neutrino detection rate.

The degree of nuclear modification can be studied as a function several variables such as nuclear size A, hadron energy fraction $z$, the square of the hadron momentum component transverse to the virtual photon direction $p_{T}^{2}$, etc. Usually the experimental results for semi-inclusive deep inelastic scattering on nuclei are presented in terms of hadronic multiplicity ratio $R_{A}^{h}$ and transverse momentum broadening, $\Delta p_{T}^{2}[1]$.

Transverse momentum broadening is defined as $\Delta p_{T}^{2}=<p_{T}^{2}>_{A}-<p_{T}^{2}>_{D}$ and is the difference of mean-square of the transverse momentum of a final state hadron produced from a larger nucleus A to that of deuterium (D) target. In certain theoretical models, this quantity is sensitive to gluonic radiation by the quark before it forms into a hadron $[3,1]$. By determining the length dependence (by varying nuclear radius) of the broadening as a function of the relevant variables, it is possible to infer the length scale (known as production length) over which the quark is de-confined.

$R_{A}^{h}$ is defined as the ratio of number of hadrons detected normalized to the number of electrons measured in the DIS kinematics with four momentum transfer $Q^{2}$ and energy transfer $v$ for a nuclear target divided by the same quantities for a deuterium target.

$$
R_{A}^{h}=\frac{\left[N_{h}\left(z, p_{T}^{2}, Q^{2}, v, \phi\right) / N_{e^{-}}^{D I S}\left(v, Q^{2}\right)\right]_{A}}{\left[N_{h}\left(z, p_{T}^{2}, Q^{2}, v, \phi\right) / N_{e^{-}}^{D I S}\left(v, Q^{2}\right)\right]_{D}}
$$

where $N_{h}$ is the number of hadrons and $N_{e^{-}}^{D I S}$ is the number of electrons produced in the specified kinematic bins. For most of the kinematic range $R_{A}^{h}$ shows a reduction of produced hadron flux in nuclei, although at low $z$ and high $p_{T}$ it shows an enhancement. Hadron formation lengths, the characteristic distances over which hadrons form, can be extracted [2] from $R_{A}^{h}$. In this paper we will concentrate on the preliminary results for $R_{A}^{h}$.

\section{Experimental details}

Previously there were several measurements to study the nuclear effects in semi-inclusive leptoproduction of hadrons. Main features observed from these data are an attenuation of hadrons which increases with A and decreases with $v$. Recently, HERMES has published multiplicity 
ratios for a variety of targets from helium to xenon [4] . For the first time they were able to measure the multiplicity ratio of several hadronic species $\left(\pi^{+}, \pi^{-}, \pi^{0}, K^{+}, K^{-}\right.$, proton and antiproton). Globally, $R_{A}^{h}$ decreases with the increasing value of the mass number. Further, there is a similarity between the data for all the pionic state. However, there is a clear difference between the kaonic charged states $\left(K^{+}\right.$being systematically larger than $\left.K^{-}\right)$.

While sophisticated models for hadronization processes exist, they are only loosely constrained by data. In order to study the multidimensional dependencies of $R_{A}^{h}$ CLAS experiment E02-104 was proposed [5] and run at Jefferson Lab during the year 2004. The CLAS data are for a lower energy range, with $2<v<5 \mathrm{GeV}$, corresponding to shorter hadron formation times. Although, CLAS data is limited in $v$ and $Q^{2}$ range, the statistical sample for pions are more than two orders of magnitude larger than HERMES. Since CLAS can accommodate solid targets this provides an opportunity to probe a series of light to heavy nuclei thus providing different length scales which is crucial for the extraction of different hadronization time scales.
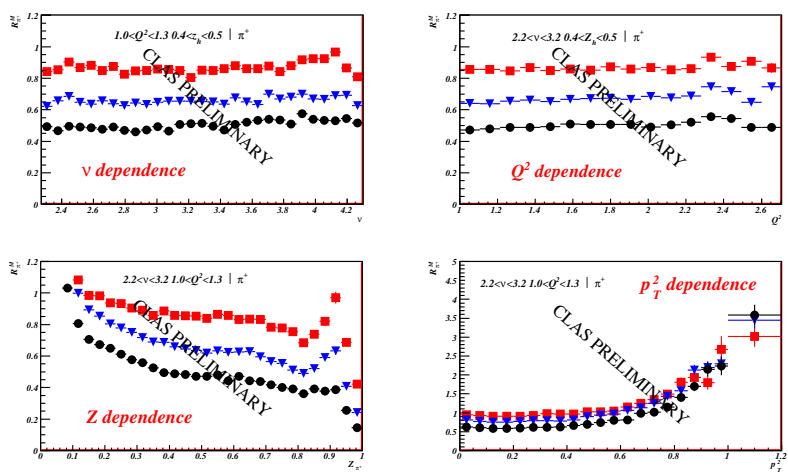

Figure 1: Preliminary CLAS data for the hadronic multiplicity ratio for positive pions (see the plot labels for the kinematic bins). Here, different colors represents different targets used in the analysis.

Several hadronic species such as $\pi^{+}, \pi^{-}, \pi^{0}, \Lambda$ and $K_{s}^{0}$ are under investigation. Although multidimensional binning was not possible for hadronic species like $K_{s}^{0}$, such binning, along with measurements of several nuclei, can be used to extract (within a given theoretical model) the hadronization time scales. Different hadron species, such as the $K_{s}^{0}$, are more relevant to study a flavor dependence of the hadronization and the quark energy loss. Thus, measurements on a variety of hadrons will provide insight into the fundamental principles of hadron formation.

These measurements were performed at Jefferson lab using $5 \mathrm{GeV}$ electron beam. The scattered electrons and produced hadrons were detected using the CLAS detector. Data was taken with deuterium "cryo" target and one of the $\mathrm{C}, \mathrm{Al}, \mathrm{Fe}, \mathrm{Sn}$ and $\mathrm{Pb}$ "solid" target simultaneously in the beam. The beam first passed through the cryo and then through a solid target. Only one solid target was exposed to the beam at a time, while all other solid targets were kept away from the beam-line using special fixtures. Since both the cryo and solid target are simultaneously exposed to the beam, most of the systematic uncertainties are expected to cancel in the target ratios. In order to select a deep inelastically scattered (DIS) electron with reduced radiative correction the following cuts were applied: $Q^{2}>1 \mathrm{GeV}^{2}, \mathrm{~W}>2 \mathrm{GeV}$ and $y=v / E<0.85$ where $\mathrm{W}$ is the invariant mass of the final state hadronic system. 
For the $K_{s}^{0}$ analysis, the $K_{s}^{0}$ was detected by reconstructing the invariant mass of a $\pi^{+} \pi^{-}$pair in coincidence with a DIS electron. The number of $K_{s}^{0}$ particles was obtained by a Gaussian fit over a smooth polynomial background. To find the $z$ dependence of the multiplicity ratios, these fits were done for both nuclear and LD2 targets, over various $z$ bins. A similar procedure was used to find the the $p_{T}^{2}$ dependence of the multiplicity ratios. It should be noted that since the number of $K_{s}^{0}$ is not large, the kinematic dependence on a given variable is extracted while integrating over other variables. Small corrections for the detection efficiency from the difference in geometry of the nuclear and $\mathrm{LD}_{2}$ targets were done with Monte Carlo simulations.

\section{Preliminary results and summary}

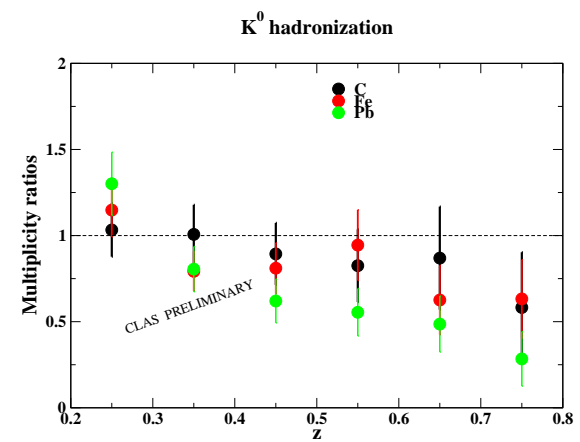

Figure 2: Preliminary CLAS data for the hadronic multiplicity ratio for $K_{s}^{0}$ summed over the kinematics of the EG2 experiment. Here, different colors represents different targets used in the analysis as shown in the legend.

Preliminary results for the $\pi^{+}$analysis [6,7] are shown in figure 1 and preliminary results for the $K_{s}^{0}$ analysis are shown in figures 2 and 3. The present data show strong attenuation effects as a function of $z$, and follow similar trends as the HERMES data for other hadrons. The lower right hand side of figures 1 and 3 show $R_{A}^{h}$ as a function of $p_{T}^{2}$. An increase of the number of hadrons at high $p_{T}^{2}$ with the atomic number $\mathrm{A}$ is observed. This effect was first observed by EMC [8] and is also known from heavy ion collisions, called the Cronin effect [9]. This effect was also observed by the HERMES collaboration [4]. Compared to experiments with heavy ions, the use of a lepton probe has the advantage that initial-state interactions do not play a role (except for shadowing effects, which are small in the $x_{B j}$ range of the HERMES and CLAS experiments). The observed rise at high $p_{T}^{2}$ might be due to a broadening of the $p_{T}^{2}$ distribution of heavy nuclei to that of deuterium. This is likely to result from partonic re-scattering as well as from hadronic final-state interactions.

To summarize, since hadronization is a non-perturbative process, only a few aspect of the process are currently understood. In particular, several hadron species are required to analyze the flavor dependence of the observables and to give insight into the fundamental principles governing hadron formation. CLAS data provides an opportunity to gain new insight into hadronization as well as other aspects of quark propagation through nuclei. Precise data for the pion channel 


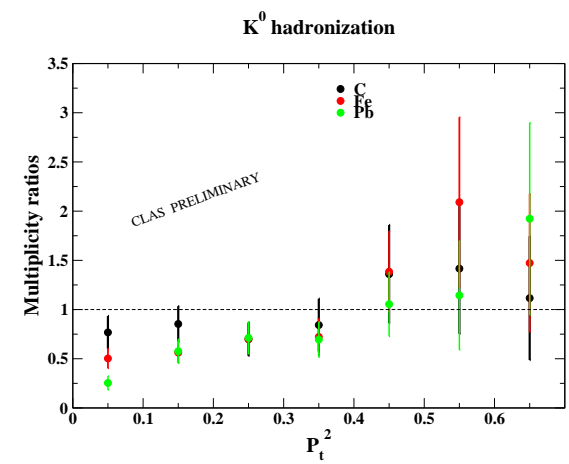

Figure 3: Results for the nuclear dependence of multiplicity ratios as a function of $p_{T}^{2}$ summed over the kinematics of the EG2 experiment. Different colors represent different targets as shown in the legend.

will provide stringent constraints on the existing models, based on different physical pictures, and will discriminate between them. Though the statistical uncertainties of the $K_{s}^{0}$ results are large, nonetheless, the preliminary data shown here are the only available data for $K_{s}^{0}$ hadronization and hence are a first step toward extending the HERMES results. The planned $12 \mathrm{GeV}$ upgrade of the Jlab and the CLAS 12 detector will offer an order of magnitude improvement in luminosity. Along with particle identification improvements, this upgrade will permit to have more precise data for several channels, and will provide further input to the efforts to understand QCD confinement in forming hadronic systems.

\section{Acknowledgments}

We would like to thank to EG2 run group for many helpful discussions and support in the analysis. This work was supported in part by NSF grant PHYS-0653454.

\section{References}

[1] A. Accardi, F. Arleo, W. K. Brooks, D. D’Enterria and V. Muccifora, Riv. Nuovo Cim. 032, 439 (2010) [arXiv:0907.3534 [nucl-th]].

[2] A. Bialas and T. Chmaj, Phys. Lett. 133B, 241 (1983).

[3] R. Baier, D. Schiff and B. F. Zakharov, Ann. Rev. Nucl. Part. Sci. 50, 37 (2000).

[4] A. Airapetian et al., Nucl. Phys. B780, 1 (2007).

[5] W. Brooks et al., Jefferson Lab Experiment E02-104, http://www.jlab.org/Hall-B/physics/experiments

[6] H. Hakobyan, Ph. D. thesis, Yeravan State University (2008).

[7] W.K. Brooks and H. Hakobyan, arXiv:0907.4606.

[8] J. Ashman et al., Z. Phys. C52, 1 (1991)

[9] J. W. Cronin et al., Phys. Rev. D11, 3105 (1975) 